\title{
CDMA coverage under mobile heterogeneous network load
}

\author{
Dorin Saban, Hans van den Berg, Richard J. Boucherie, Irwan Endrayanto \\ Stochastic Operations Research Group, Faculty of Mathematical Sciences \\ University of Twente, P.O. Box 217, 7500 AE Enschede, The Netherlands \\ tel. +31-53-4892410, fax. +31-53-4894858, e-mail d.saban@math.utwente.nl
}

\begin{abstract}
We analytically investigate coverage (determined by the uplink) under non-homogeneous and moving traffic load of third generation UMTS mobile networks. In particular, for different call assignment policies we investigate cell breathing and the movement of the coverage gap occurring between cells when a hot spot moves among the cells. These call assignment policies mainly differ in handling non feasible call configurations. To establish the maximally possible coverage, calls at the cell borders will be dropped such that the remaining carried calls establish their SIR target. By assigning calls to different base stations according to these policies, the coverage gap differs especially under moving non-homogeneous load.
\end{abstract}

\section{INTRODUCTION}

The third generation UMTS network air interface is based on the flexible and efficient CDMA multiplexing technique that enables all calls to use the entire bandwidth. As a consequence, users compete for power in the allocated bandwidth and a keyfactor for analysis becomes the interference level. The problem of minimization of total interference in the network (or in other words maximization of network capacity) remains one of the major research topics in the field of DS-WCDMA. This problem has mainly two complimentary parts: (1) power control, and (2) assignment of calls to base stations. In the analysis of the second part it is sometimes overlooked that optimality of some specific assignment strategies depends not only on nonhomogeneity of traffic load between cells but also on distribution of this load within the cells. Knowledge of the call distribution helps to choose an optimal assignment for a specific situation and gives the opportunity to optimize the available channel space.

It is commonly agreed that (due to the asymmetrical nature of data traffic) the capacity of the CDMA system is determined by the downlink, but coverage is determined by the uplink [8]. The present study analytically investigates coverage under nonhomogeneous and moving teletraffic load, and therefore focusses on the uplink. In particular, for different call assignment policies we investigate cell breathing and the movement of the coverage gap occurring between cells when a hot spot moves among the cells. The different call assignment strategies considered in this paper are characterized by the following optimization objectives: (a) minimal total received power, (b)

This research is partially is supported by the Technology Foundation STW, applied science division of NWO and the technology programme of the Ministry of Economic Affairs, The Netherlands. minimal total transmitted power, (c) minimal total interference. For comparison we also consider the situation with a fixed border in the middle between base stations. Our analysis is set up for a general network configuration. For a simplified, yet representative, two cell network we provide numerous numerical results, which illustrate the performance of the different call assignment strategies under various traffic loads.

The rest of this paper is organized as follows. In the next section, we discuss some related literature. Section III provides a brief description of the modelling approach used in this paper. In Section IV-VI we give a mathematical description of the optimization problems to be solved and derive and discuss some useful properties. Section VII contains the numerical results for the simplified two cell network. Finally, in Section VIII, we summarize and conclude our study.

\section{RELATED LITERATURE}

A substantial body of literature focusses on the CDMA uplink. However, most references consider either homogeneously loaded cells or non moving calls. An important exception is [14], where (via simulation) for non homogeneous but non moving load the capacity (number of carried calls) is considered. The coverage, however, is not taken into account.

A nice overview of distributed power control algorithms that minimize total transmitted power is provided in [8]. Recent references on optimal power control are e.g. [12] and [10]. In particular, in [10] the impact of the statistical variations of the signals due to fading are considered, and the authors show that well-known methods for allocating power (which ignore fading; often based on Perron-Frobenius eigenvalue theory) can be used to determine power allocations that are close to achieving optimal (i.e. minimal) outage.

Call assignment strategies are presented in e.g. [2], [7], [1], [3] and [6]. In particular, in [2] several call dropping strategies to be applied in case of congestion (and leading to coverage gaps) are discussed and evaluated. Their analysis is based on simulations. The authors of [1] also study and compare different dropping strategies for situations in which not all SIR targets can be attained. They provide an analytical study for homogeneous traffic loads and nicely point out the unfavorable effects that may occur for certain dropping strategies. 
The focus of this paper is to derive a method to analytically investigate coverage of UMTS networks. Therefore, a simplified network is defined by a set of base station positions, a spatial user distribution, and a deterministic pathloss model that takes into account only the distance between mobiles and base stations. The addition of random components such as shadowing will not qualitatively alter the results, as the resulting randomness can be taken into account as a change of the location of the subscribers cf. [13]. Macro diversity is not incorporated in our model. We further assume perfect power control.

We assume that all mobiles use the same service with the same constant bit rate, and therefore have the same SIR target. We focus on data calls, so that transmission below the SIR target will result in the number of dropped packets exceeding the error correction capability of the error correction code (which is the nature of the SIR target). Therefore, reducing the SIR target for all mobiles (soft capacity) dramatically reduces the performance of the network, and it seems better to (temporarily) drop some mobiles. Thus, in our study we investigate call dropping due to coverage problems occurring as a consequence of heterogeneous network load.

We do not take into account the randomness of the call arrival and termination process. As is shown in [15], this randomness can be transformed into required capacity, so that for our purpose of investigating the impact of heterogeneous load on coverage it suffices to use a deterministic user pattern. The examples of Section VII numerically reflect our findings for a simple two cell linear network representing a highway, along which traffic moves in one direction. We investigate inter and intra cellular interference, and focus on the coverage gap occuring when hot spots travel along the road. Coverage of a base station is defined via the location of mobiles handled by the base station. As a consequence, due to granularity of the distribution of mobiles, there may seem to be a gap in the coverage when neighbouring mobiles are connected to different base stations, see e.g. Figure 1. This gap will be referred to as granularity gap. A coverage gap occurs when mobiles are dropped due to their SIR requirement: a coverage gap will contain a number of mobiles, and all mobiles not contained in the gap establish their SIR target.

Under the call assignment strategies (a) minimal total received power, (b) minimal total transmitted power, (c) minimal total interference, the size of the coverage gap (number of mobiles in the gap) is obviously the same, and depends only on the density of mobiles. In a practical implementation, where assigned calls travel along a road, these strategies may result in considerably different behaviour, when among the already accepted calls some have to be dropped due to their constraint on maximal transmission power while moving away from a base station. To represent this dynamic behaviour of the call assignment strategies, we first establish the feasible configurations not taking into account the maximum on transmitted power of mobiles (e.g. the hot spot is close to a base station), and then investigate which mobiles have to be dropped when taking into account this additional constraint.
Consider a WCDMA system containing $K$ base stations and $N$ calls. Let $N_{k}$ denote the number of calls assigned to base station $k, k=1, \ldots, K$. In our uplink model, the received power at base station $k$ is denoted $P_{k}$, and assumed equal for all mobiles power controlled by base station $k$. Let $h_{i k}$ denote the path gain from mobile $i$ to base station $k$, i.e., mobile $i$ received at base station $k$ with power $P_{k}$ is transmitting with power $P_{k} / h_{i k}$. The Quality of Service (QoS) for a mobile power controlled by base station $k$ is then determined by the carrier to interference ratio $(C / I)$, for $k=1, \ldots, K$ :

$$
\left(\frac{C}{I}\right)_{k}=\frac{W P_{k}}{\left(N_{k}-1\right) P_{k}+\sum_{\ell \neq k} \sum_{i=1}^{N_{\ell}} \frac{h_{i k}}{h_{i \ell}} P_{\ell}+W \eta_{k}},
$$

where $W$ denotes the processing gain, and $\eta_{k}$ is the power of the thermal noise. Clearly, the assignment of mobiles to base stations as reflected in the variables $N_{k}$, and the location of the mobiles as reflected in the path gains $h_{i k}$ play an important role in this power control equation.

We assume that the required QoS is equal for all mobiles. Let $\varepsilon=\left(\frac{C}{I}\right)_{k}$ denote the target QoS requirement, equal for all base stations. In a network consisting of a single base station, a feasible power assignment exists only if

$$
\varepsilon \leq \frac{W P}{(N-1) P+W \eta},
$$

or equivalently only if the required power $P \leq P_{\text {pole }}$, where

$$
P_{\text {pole }}=\frac{W \eta}{1-N+W / \varepsilon}
$$

which requires that $N \leq N_{\text {pole }}=1+W / \varepsilon$, where $N_{\text {pole }}$ is the pole capacity of a single base station. In addition, the power budget of mobiles, $p_{\max }$, is limited, resulting in the restriction

$$
P_{k} / h_{i k} \leq p_{\max }
$$

For a multi cell network, let $c[i]$ denote the base station mobile $i$ is assigned to under a power assignment policy, $i=$ $1, \ldots, N$, and $c=(c[1], \ldots, c[N])$. Then $N_{k}=\sum_{i} 1(c[i]=k)$, where $1($.$) denotes the indicator function. As a power assign-$ ment accomodating all mobiles may not be feasible, we have $\sum_{k} N_{k} \leq N$. An assignment of mobiles to base stations is feasible if and only if

$$
\left\{\begin{array}{l}
M(\mathbf{N}) \mathbb{P}=R \\
P_{c[i]} / h_{i c[i]} \leq p_{\max }, \quad i=1, \ldots, \sum_{k} N_{k}
\end{array}\right.
$$

where $\mathbf{N}=\left(N_{1}, \ldots, N_{K}\right), \mathbb{P}=\left(P_{1}, \ldots, P_{K}\right)$, $R=\left(W \eta_{1}, \ldots, W \eta_{K}\right)$, and the $K \times K$ matrix $M(\mathbf{N})$ is given by

$$
M[k, \ell]= \begin{cases}-L_{\ell, k}=-\sum_{i=1}^{N_{\ell}} \frac{h_{i k}}{h_{i \ell}} & k \neq \ell \\ N_{k}-1-W / \varepsilon & k=\ell\end{cases}
$$

Notice that for feasibility it must be that $N_{k} \leq N_{\text {pole }}=1+$ $W / \varepsilon, k=1, \ldots, K$. We will denote $\mathbb{P} \in \mathbb{F}$ when $\mathbb{P}$ satisfies (5). 
The maximum number of carried calls is determined as maximizing argument, $c[i], i=1, \ldots, N$, of the following optimization problem

$$
\max _{c[i], i=1, \ldots, N} \sum_{k} N_{k} \quad \text { s.t } \quad \begin{aligned}
& N_{k}=\sum_{i} 1(c[i]=k) \\
& \mathbb{P} \in \mathbb{F}
\end{aligned}
$$

Let

$$
\mathbb{C}=\arg \max _{c[i], i=1, \ldots, N} \sum_{k} N_{k} \quad \text { s.t } \begin{aligned}
& N_{k}=\sum_{i} 1(c[i]=k) \\
& \mathbb{P} \in \mathbb{F}
\end{aligned}
$$

The maximizing argument need not be unique, i.e., multiple distributions of mobiles to base stations may yield the same number of carried calls. Among these configurations, the following power assignment algorithms select an optimal assignment.

\section{FeAsible POWER ASSIGNMENTS}

Selection of the optimal power assignment among the feasible assignments determined by (7) requires a criterion for optimality. Clearly, the first criterion as determined by (6) is to carry as many calls as possible. Then, within the set $\mathbb{C}$ assignment of calls to different cells may be carried out optimally according to various power assignment criteria, see Section II for methods used in literature. Below we collect three call assignment protocols based on the maximum number of carried calls determined by $\mathbb{C}$. In addition, for comparison, we also investigate a UMTS network of cells with fixed borders located in the middle between base stations.

\section{a) Minimal received power}

The power assignment that minimizes the total received power $P^{r e c}=\sum_{\ell} P_{\ell}$ is determined as

$$
\arg \min _{c \in \mathbb{C}} \sum_{\ell=1}^{K} P_{\ell}
$$

\section{b) Minimal transmitted power}

The power assignment that minimizes the total transmitted power $P^{\text {trans }}=\sum_{i=1}^{N} \frac{P_{c[i]}}{h_{i c[i]}}$ is determined as

$$
\arg \min _{c \in \mathbb{C}} \sum_{i=1}^{N} \frac{P_{c[i]}}{h_{i c[i]}}
$$

\section{c) Mimimal total interference}

The interference relation between base stations $k$ and $\ell$ due to mobile $j$ can be expressed as the ratio of received powers due to mobile $j$ transmitting at unit power: $\frac{P_{k}}{P_{\ell}} \frac{h_{\ell, j}}{h_{k, j}}$. Clearly, the tradeoff in assigning a mobile to either base station $k$ or $\ell$ can be made on the basis of this interference relation, or on the symmetrical version $\frac{P_{k}}{P_{\ell}} \frac{h_{\ell, j}}{h_{k, j}}+\frac{P_{\ell}}{P_{k}} \frac{h_{k, j}}{h_{\ell, j}}$. The total interference then takes into account all mobiles. Thus, the power assignment that minimizes total interference is determined as

$$
\arg \min _{c \in \mathbb{C}} \sum_{i=1}^{N} \sum_{\ell=1, \ell \neq c[i]}^{K}\left(\frac{P_{c[i]}}{P_{\ell}} \frac{h_{i \ell}}{h_{i c[i]}}+\frac{P_{\ell}}{P_{c[i]}} \frac{h_{i c[i]}}{h_{i \ell}}\right)
$$

It is interesting to note that for a feasible assignment our minimal total interference criterion coincides with the criterion used in [7], where feasibility is characterised via the PerronFrobenius eigenvalue of the $K \times K$ interference matrix $\mathcal{A}$ with entries $\mathcal{A}[\ell, k]=\frac{\epsilon}{W} \frac{N_{\ell}}{N_{k}} L_{k, \ell}$.

\section{d) Fixed border}

For comparison, we also provide a characterisation of the optimal assignment under a fixed border that is located in the middle between base stations. This requirement means that $h_{i c[i]} \leq h_{i k}$ for all $k \neq c[i]$. Let us consider minimization of received power. The power assignment that minimizes the total received power $P^{r e c}=\sum_{\ell} P_{\ell}$ is determined as

$$
\arg \min _{c \in \mathbb{C}_{f}} \sum_{\ell=1}^{K} P_{\ell}
$$

where $\mathbb{C}_{f}=\mathbb{C}$ with the additional constraints $h_{i c[i]} \leq h_{i k}$ for all $k \neq c[i]$.

\section{POWER ASSIGNMENT ALGORITHMS}

A practical call or power assignment does not re-evaluate the feasible call configuration to accept non carried calls in favour of carried calls (i.e. drops calls to carry new calls). Instead, a practical implementation will attempt to carry all existing calls, and drops existing calls only in favour of other carried calls to meet their SIR requirements. To investigate the behaviour of the power assignments described above, we investigate their behaviour for moving calls. In particular, we will start with a feasible assignment not taking into account the power limitation $p_{\max }$ of the mobiles (which mimics the behaviour when a hot spot is not too far from the base station). When the hot spot moves away from the base station, the mobiles will increase their transmitted power until they reach $p_{\max }$, and subsequently some mobiles will no longer be able to reach the required transmission power. When this happens, from the carried calls some calls will be dropped. Obviously, the resulting call assignment is sub-optimal, which can easily be seen from a mathematical consideration of the procedure (recall (5)): We first consider an assignment of mobiles under the constraint $M \mathbb{P}=R$, only. Let $\mathbb{C}_{p}$ denote the corresponding solution of (7) with $\mathbb{P} \in \mathbb{F}$ replaced by $M \mathbb{P}=R$. Obviously $\mathbb{C} \subset \mathbb{C}_{p}$. Now, among the assignments of $\mathbb{C}_{p}$ select the optimal feasible assignments under the additional requirement $P_{c[i]} / h_{i c[i]} \leq p_{\max }, \quad i=1, \ldots, \sum_{k} N_{k}$. If this assignment exists, then it is feasible for (6). If it does not exist, then drop the call with largest $P_{c[i]} / h_{i c[i]}$ and re-evaluate the resulting call configuration. Clearly, the resulting assignment is sub-optimal under (6).

The sub-optimal call and power assignment introduced above can be done based on local information. To this end, consider the power assignment equation $M(\mathbf{N}) \mathbb{P}=R$. When we shift mobile $r$ from cell $k=c[r]$ to cell $\ell$ feasibility of the assignment is determined by the power assignment equation $M\left(\mathbf{N}^{\prime}\right) \mathbb{P}^{\prime}=R$, where $\mathbf{N}^{\prime}=\left(N_{1}, \ldots, N_{i}-1, \ldots, N_{j}+\right.$ 
Coverage gap under (6), rectangular peak

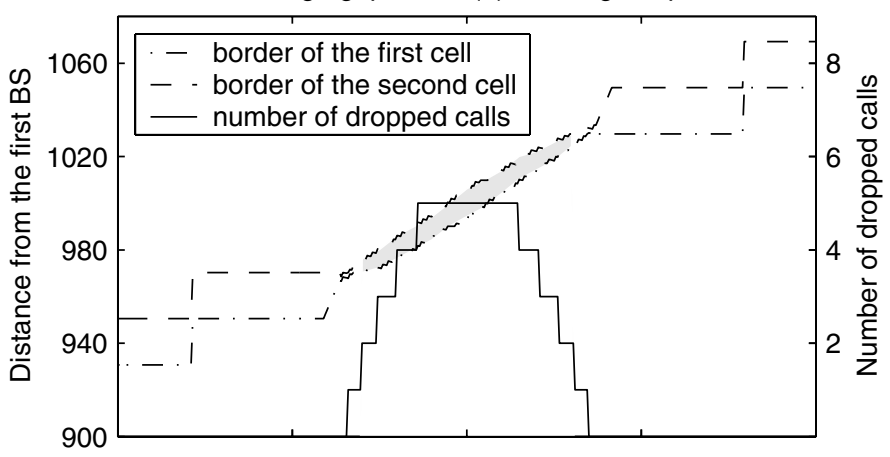

Coverage gap under (6), triangular peak

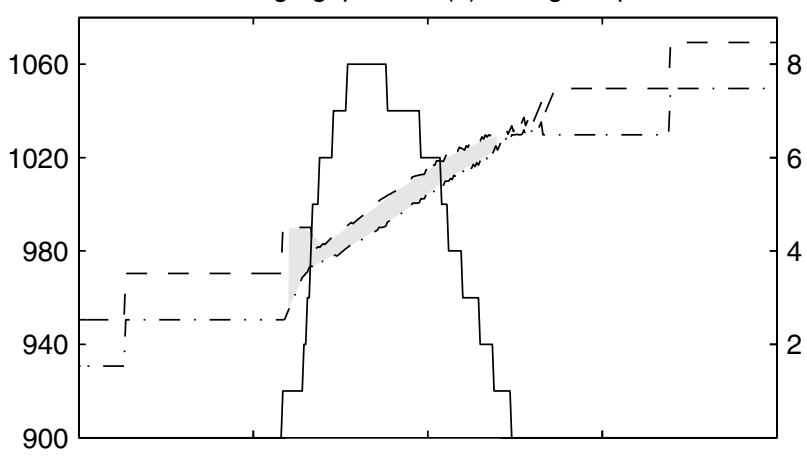

Fixed borders, rectangular peak

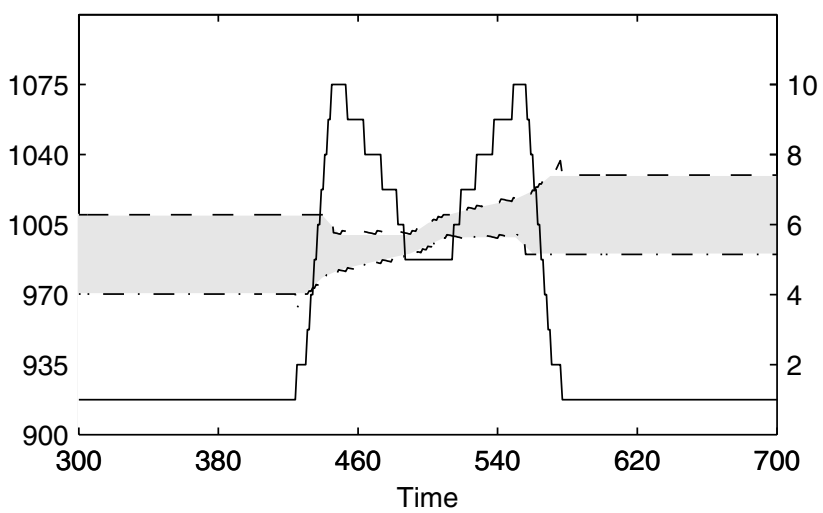

Fig. 1. Coverage gap under the optimal (centralized) call and power assignment determined by (6) and in case of fixed borders

$\left.1, \ldots, N_{K}\right)$. Let $\Delta \mathbb{P}=\mathbb{P}^{\prime}-\mathbb{P}$, then substracting the power assignment equations for $\mathbf{N}^{\prime}$ and $\mathbf{N}$ yields

$$
M\left(\mathbf{N}^{\prime}\right) \Delta \mathbb{P}=\left(\frac{h_{r k}}{h_{r \ell}} \frac{P_{\ell}}{P_{k}}-1\right) P_{k} H(r),
$$

where $H(r)=\left(\frac{h_{r 1}}{h_{r k}}, \ldots, \frac{h_{r K}}{h_{r k}}\right)$. Under the assumption that a feasible power assignment exists for $\mathbf{N}^{\prime}$, the sign of $\left(\frac{h_{r k}}{h_{r \ell}} \frac{P_{\ell}}{P_{k}}-1\right)$ now determines the sign of $\Delta \mathbb{P}$. As a consequence, if $\frac{h_{r k}}{h_{r l}} \frac{P_{\ell}}{P_{k}}<1$ then the new assignment obtained by shifting mobile $r$ to base station $\ell$ results in a lower received power for all base stations (and a lower transmitted power for all mobiles). Thus, for minimization of transmitted power it
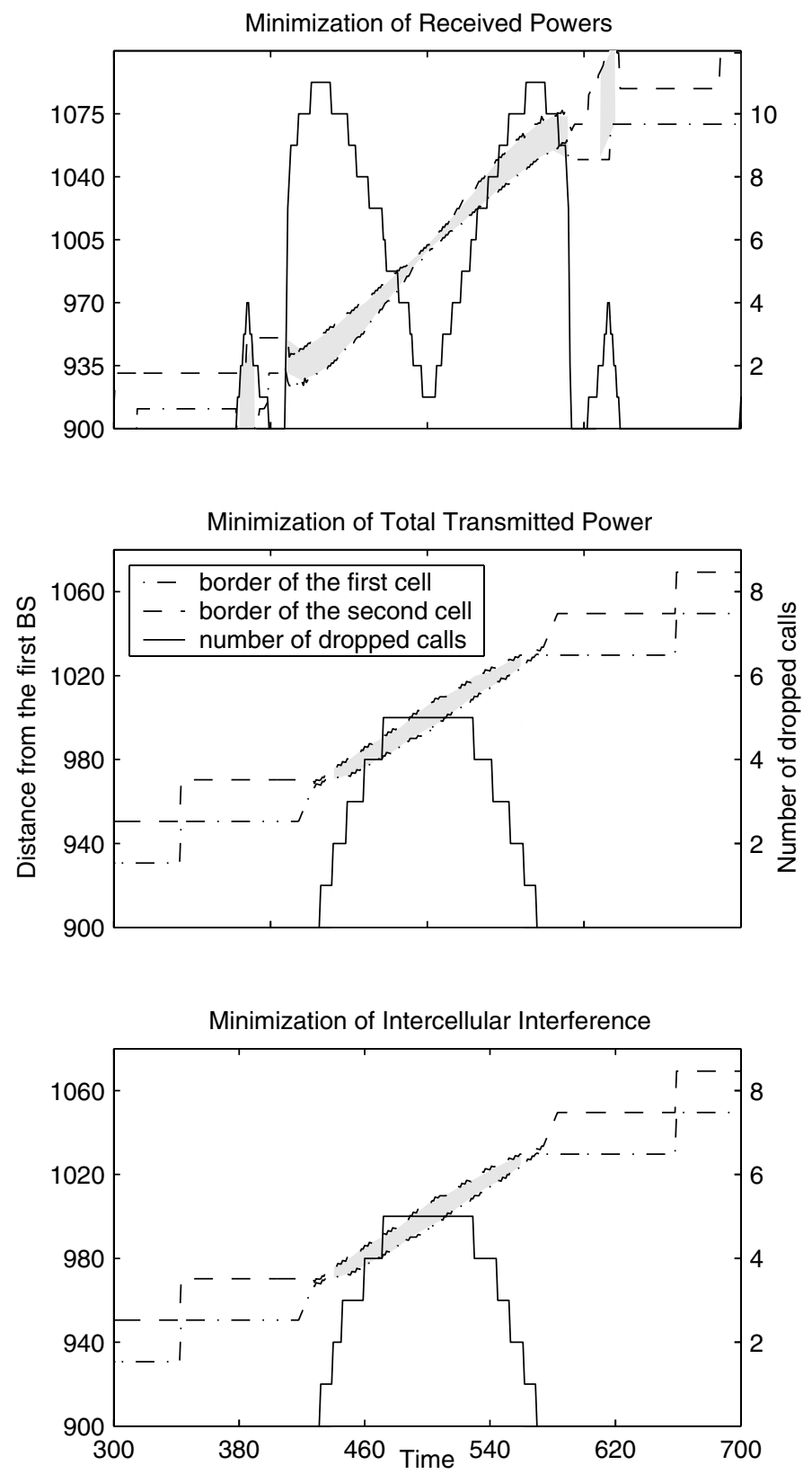

Fig. 2. Coverage gap resulting from moving rectangular peak, under the suboptimal (distributed) call and power assignment strategies introduced in Section V-VI.

suffices to compare an assignment of mobiles with an assignment with one mobile shifted to a neighbouring base station to optimize transmitted power, resulting in a distributed implementation of power optimization. Similarly, observing that $\frac{P_{c[i]}}{P_{\ell}} \frac{h_{i \ell}}{h_{i c[i]}}+\frac{P_{\ell}}{P_{c[i]}} \frac{h_{i c[i]}}{h_{i \ell}}$ is a convex function of $c[i]$, that attains its minimum for $\frac{P_{c[i]}}{P_{\ell}} \frac{h_{i \ell}}{h_{i c[i]}}=1$, we see that comparing assignments $c$ and $c^{\prime}$ can be done in a distributed implementation, too. Thus, ignoring the power limitation $p_{\max }$, the global (or centralised) power assignments of mimimizing transmitted power or minimizing total interference allow a decentralised implementation. 


\section{NUMERICAL RESULTS}

To illustrate the coverage analysis described in the previous sections, in this section we will present extensive numerical results for a simple two cell linear network. The traffic carried by the network consists of a fixed number of background calls homogeneously distributed over the two cells and a peak (hot spot) moving at constant speed from the left base station to the right one.

In our numerical examples we have considered traffic peaks with two different shapes: a rectangle and an orthogonal triangle with 90 degree angle on the right hand side. The values of the system parameters used in our example are as follows. The distance between the two base stations is 2000 meters. The standard WCDMA chip rate $(3.84 \mathrm{Mc} / \mathrm{s})$ has been used and we have taken the thermal noise $\eta=-169 \mathrm{dBm} / \mathrm{Hz}$; the path gain exponent is equal to -4 . The background traffic and the traffic peaks each consist of 100 calls; the traffic peaks have a basis of 200 meters. Each call generates traffic at a fixed rate of 12.2 Kbit/s. The $C / I$ target $\epsilon=3 \mathrm{dBm}$.

We have first (numerically) solved the global optimal call and power assignment problem (6) such that the number of carried calls is maximized at each moment in time. Figure 1 shows the resulting movement of the cell borders (i.e. distance to left base station) as a function of time. The coverage gap is indicated in the figures by the gray area, where the cell borders diverge. The number of calls that is dropped in the coverage gap is shown by the solid curve scaled along the right $y$-axis. Note, that the 'gaps' between the cell borders outside the gray area are due to the granularity of the distribution of mobiles over the cells (there is no call dropping, cf. the discussion in Section III). Comparison of the figures for triangular and rectangular traffic peaks reveals that for the triangular peak the coverage gap (number of dropped calls) increases more rapidly than for the rectangular peak, and, after having reached its maximum, it also decreases faster than the gap resulting from the rectangular peak. Obviously, this is due to the 'smoother' shape of the rectangle. The total number of dropped calls for the triangular peak is much larger than for the rectangular peak. For comparison, the coverage results for the rectangular traffic peak in the case of a fixed cell border in the middle between the two cells is depicted in the last graph of Figure 1. As expected, the coverage gap is considerably larger than in the previous example with a moving border ('cell breathing').

Figure 2 shows the coverage gap (for moving rectangular traffic peak) resulting from the sub-optimal distributed call and power assignment procedure described in Section VI, for three different criteria. The results indicate that, for our parameter settings, minimization of transmitted power and intercellular interference in a distributed approach are close to optimality.

\section{CONCLUDING REMARKS}

The main contribution of this paper is the analytical characterization of the coverage gap in a CDMA network (uplink) resulting from moving hot spots. To investigate coverage and capacity, we have introduced and compared power and call assignment (to base stations) policies that minimize the coverage gap under different additional constraints. The optimal assignment policy requires a centralized approach, which may be too complex for implementation in real networks. Therefore, we have also investigated a (sub-optimal) distributed approach, in which decisions about call dropping are based on locally available information on the status of the network. The assignment policies have been numerically evaluated for a simplified two cell linear network representing e.g. a highway. The numerical results confirm our expectations concerning the occurrence of the coverage gap, and illustrate the differences (with respect to e.g. the size and 'shape' of the coverage gap) between the various power and call assignment policies. In particular, in our example some of the sub-optimal distributed approaches are only slightly less efficient than the centralized, optimal power assignment strategy.

More numerical results (for more general settings of the network and traffic load) have to be obtained in order to be able to derive final conclusions about the performance of the various power and call assignment policies. This is our first objective for further research. Another issue for further research is call admission control (CAC). Our results may be applicable to develop enhanced CAC algorithms that take into account not only immediate consequences of the assignment of (new) calls to a particular base station but also the possible future positions of the calls. Finally, we also intend to extend our present work on uplink coverage to coverage in the downlink.

\section{REFERENCES}

[1] M. Andersin, Z. Rosberg, and J. Zander, Gradual Removals in Cellular PCS with Constrained Power Control and Noise, Wireless Networks, Vol. 2, (1996), 27-43.

[2] L. L. H. Andrew, S. V. Hanly, Performance of a Global Congestion Measure for CDMA Networks, Proc. Vehicular Technology Conference 1999Fall, Amsterdam, The Netherlands, 1999.

[3] S. C. Chen, N. Bambos, G. J. Pottie, Admission Control Schemes for Wireless Communication Networks with Adjustable Transmitter Powers. INFOCOM 1994: 21-28.

[4] A. Ghosh and R. Love, "Mobile Station Location in a DS-CDMA System”, presented at VTC98, pages 254-258. Motorola, 1998.

[5] S. Grandhi, J. Zander, and R. Yates. Constrained power control. International Journal of Wireless Personal Communications 1(4), 1995.

[6] Hanly S.V. (1995) “ An algorithm for combined cell-site selection and power control to maximize cellular spread spectrum capacity." IEEE Journal on Selected Areas in Communications, Vol. 13, No. 7, September: 1332-1340.

[7] Hanly S.V. (1999) "Congestion measures in DS-CDMA networks ", IEEE Transactions on Communications, Vol. 47, No. 3, March:426-437.

[8] S.V. Hanly, D.N. Tse, Power control and capacity of spread spectrum wireless networks, Automatica 35 (12), pp. 1987-2012, 1999 AddisonWesley, 1998.

[9] H. Holma, H., A. Toskala, WCDMA for UMTS, Revised edition, Wiley, 2001.

[10] S. Kandukuri and S. Boyd, Optimal power control in interference limited fading wireless channels with outage probability specifications IEEE Journal on Wireless Communications, Volume 1, Number 1, pages 46-55, January 2002.

[11] K. Leibnitz, Impacts of Power Control on Outage Probability in CDMA Wireless Systems. Broadband Communications 1999: 225-234.

[12] L. Qian and Z. Gajic, "Optimal Distributed Power Control in Cellular Wireless Systems", 38th Annual Allerton Conference on Communication, Control, and Computing, Vol.1, pp.393-402,Urbana, IL, Oct 2000.

[13] Lei Song, N.B. Mandayam, and Z. Gajic, Analysis of an Up/Down Power Control Algorithm for the CDMA Reverse Link under Fading. IEEE JSAC Wireless Series, vol. 19, No. 2, pp. 277-286, February 2001.

[14] D. Staehle, K. Leibnitz, K. Heck, B. Schröder, A. Weller, and P. TranGia, "Approximating the Othercell Interference Distribution in Inhomogeneous UMTS Networks", Proc. of the IEEE Vehicular Technology Conference (VTC-02 Spring), Birmingham, AL, May 2002.

[15] A. Ule, R.J. Boucherie (2000) Adaptive dynamic channel borrowing in road-covering mobile networks, Memorandum No. 1589, Faculty of Mathematical Sciences, University of Twente, 2001. 